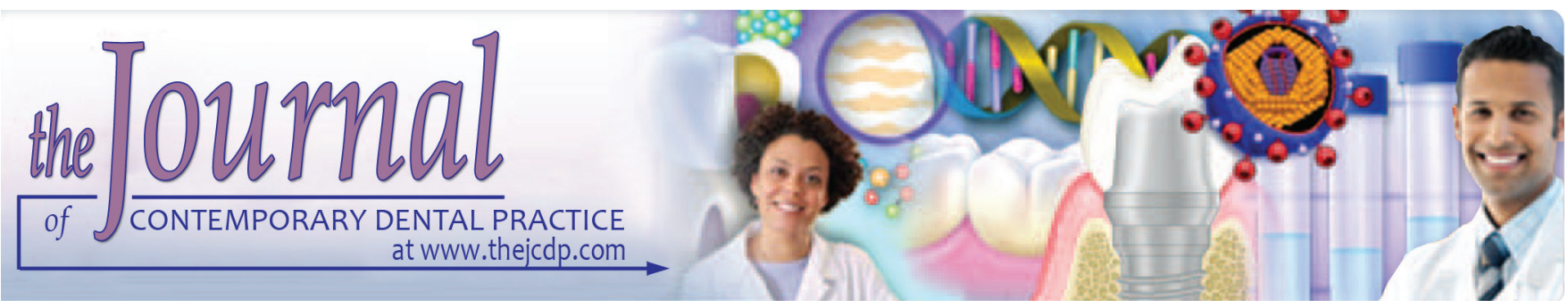

\title{
Assessment of Oral Status in Pediatric Patients with Special Health Care Needs receiving Dental Rehabilitation Procedures under General Anesthesia: A Retrospective Analysis
}

\author{
${ }^{1}$ Neeraj Solanki, ${ }^{2}$ Anuj Kumar, ${ }^{3}$ Neha Awasthi, ${ }^{4}$ Anjali Kundu, ${ }^{5}$ Suveet Mathur, ${ }^{6}$ Suresh Bidhumadhav
}

\begin{abstract}
Introduction: Dental problems serve as additional burden on the children with special health care needs (CSHCN) because of additional hospitalization pressure, they face for the treatment of various serious medical problems. These patients have higher incidence of dental caries due to increased quantity of sugar involved in the drug therapies and lower salivary flow in the oral cavity. Such patients are difficult to treat with local anesthesia or inhaled sedatives. Single-sitting dental treatment is possible in these patients with general anesthesia. Therefore, we conducted this retrospective analysis of oral health status of CSHCN receiving various dental treatments in a given population.
\end{abstract}

Materials and methods: A total of $200 \mathrm{CSHCN}$ of age 14 years or less reporting in the pediatric wing of the general hospital from 2005 to 2014 that underwent comprehensive dental treatment under general anesthesia were included in the study. Patients with history of any additional systemic illness, any malignancy, any known drug allergy, or previous history of any dental treatment were excluded from the study. Complete mouth rehabilitation was done in these patients under general anesthesia following standard protocols. Data regarding the

${ }^{1}$ Department of Pedodontics and Preventive Dentistry, Kalka Dental College \& Hospital, Meerut, Uttar Pradesh, India

${ }^{2}$ Department of Maxillofacial Surgery, Bhagwan Mahavir Medica Superspecialty Hospital, Ranchi, Jharkhand, India

${ }^{3}$ Department of Pedodontics and Preventive Dentistry, School of Dental Sciences, Sharda University, Greater Noida, Uttar Pradesh, India

4,5 Department of Oral and Maxillofacial Surgery, Vyas Dental College \& Hospital, Jodhpur, Rajasthan, India

${ }^{6}$ Private Practice, Bengaluru, Karnataka, India

Corresponding Author: Neeraj Solanki, Reader, Department of Pedodontics and Preventive Dentistry, Kalka Dental College \& Hospital, Meerut, Uttar Pradesh, India, Phone: +919414509596, e-mail: drneerajsolanki@gmail.com patient's disability, type, duration, and severity of disability was collected and analyzed. All the results were analyzed by Statistical Package for the Social Sciences (SPSS) software. Chi-square test, Student's t-test, and one-way analysis of variance were used to assess the level of significance.

Results: Statistically significant results were obtained while analyzing the subject's decayed missing filled/decayed extracted filled teeth indices divided based on age. Significant difference was observed only in cases where patients underwent complete crown placement even when divided based on type of disability. While analyzing the prevalence, statistically significant results were observed in patients when divided based on their age.

Conclusion: In CSHCN, dental pathologies and caries indices are increased regardless of the type or extent of disability.

Clinical significance: Children with special health care needs should be given special oral health care, and regular dental checkup should be conducted as they are more prone to have dental problems.

Keywords: Anesthesia, Children with special health care needs, Dental care, Disability.

How to cite this article: Solanki N, Kumar A, Awasthi N, Kundu A, Mathur S, Bidhumadhav S. Assessment of Oral Status in Pediatric Patients with Special Health Care Needs receiving Dental Rehabilitation Procedures under General Anesthesia: A Retrospective Analysis. J Contemp Dent Pract 2016;17(6):476-479.

Source of support: Nil

Conflict of interest: None

\section{INTRODUCTION}

Caries is one of the commonest dental problems worldwide. Children with special health care needs (CSHCN) have increased burden of such dental problems since they have been already exposed to intense hospitalization for various serious medical problems. American Academy 
of Pediatric Dentistry in 2004 have defined special health care needs as conditions that "include any physical, developmental, mental, sensory, behavioral, cognitive, or emotional impairment or limiting condition that requires medical management, health care intervention, and/or use of specialised services or programs. The condition may be developmental or acquired and may cause limitations in performing daily self-maintenance activities or substantial limitations in a major life activity."1 Because of presence of increased quantity of sugar in the medicinal therapy, and due to lower cleansing action by saliva, CSHCN are at elevated risk of development of orodental pathologies., ${ }^{2,3}$ Absence of specialized treatment modalities as well as dental specialists and lack of concern toward the dental treatment of guardians of these patients, due to ongoing treatment for systemic problems, are the two major issues that are encountered by CSHCN from the oral health's prospective. ${ }^{4}$ With local anesthesia alone or along with inhaled sedatives, the CSHCN are unable to tolerate the dental rehabilitation. Single-sitting dental treatments, such as restorative procedures, root canal therapies, and crown fabrications, etc., of such patients are possible when treatment is carried out in general anesthesia. ${ }^{5}$ Therefore, we conducted this retrospective analysis of oral health status of CSHCN receiving various dental treatments in a given population.

\section{MATERIALS AND METHODS}

The present study was carried out in the pediatric wing of the general hospital. A total of $200 \mathrm{CSHCN}$ of age 14 years and less reporting in the pediatric wing from 2005 to 2014 that underwent comprehensive dental treatment under general anesthesia were included in the study. Ethical approval was taken from the Institution's Ethical Committee and written consent was obtained from the parents/guardians of the patients by preinforming them about the study protocol. For the evaluation of the psychological, physical, and behavioral capabilities, initial examination of all the subjects was done by experienced pediatricians and pediatric dental surgeons. Only those patients were included who had negative history of any previous dental treatment. Patients with any other systemic illness, metabolic disorder, any known drug allergy, or with history of any previous dental treatment were excluded from the study. Skilled pediatricians and anesthesiologists were consulted before the treatment planning to assess the systemic factors contraindicated during the general anesthesia procedure. Complete mouth rehabilitation was done in these patients following standard protocols. For treating pits and fissures of the teeth, fissure sealants were used. In teeth with multiple, deep caries, root canal procedures were carried out followed by crown fabrications. In teeth where rehabilitation by restoration was not possible, extraction was done. For periodic checkup, all the patients were recalled after every 3 months. Approximately at the age of 12 , when patients' permanent dentition reached occlusal level, assessment of malocclusion was done. Anterior and posterior deep bite, ectopic eruption, crossbite, malaligned teeth, etc. were the parameters considered for categorizing malocclusion. Complete data about patient's disability, type, duration, and severity of disability was collected and analyzed. Patient's National Disability certificate was used to evaluate the type of medical problem. Various disabilities included autism, mental retardation, disability of the limbs, vision irregularity, speech defect, primary organ's function loss, and defect in balancing ability. Decayed missing filled (DMF) and decayed extracted filled (DEF) index were evaluated based on WHO criteria. Combination of the abovementioned two indices gave us the total caries present. All the patients were divided based on the age groups to calculate the results. Malocclusion was also recorded and assessed. All the results were analyzed by SPSS software. Chi-square test, Student's t-test and one-way analysis of variance were used to assess the level of significance.

\section{RESULTS}

Graph 1 highlights the various demographic details of the patients included in the study. While analyzing the DMF/DEF teeth indices, statistically significant ( $p$-value $<0.05$ ) results were obtained only in patients groups that were divided based on age (Table 1). While comparing the distribution of dental treatment by various treatment modalities divided based on the type of disability, we observed significant difference only in cases of patients who underwent complete crown

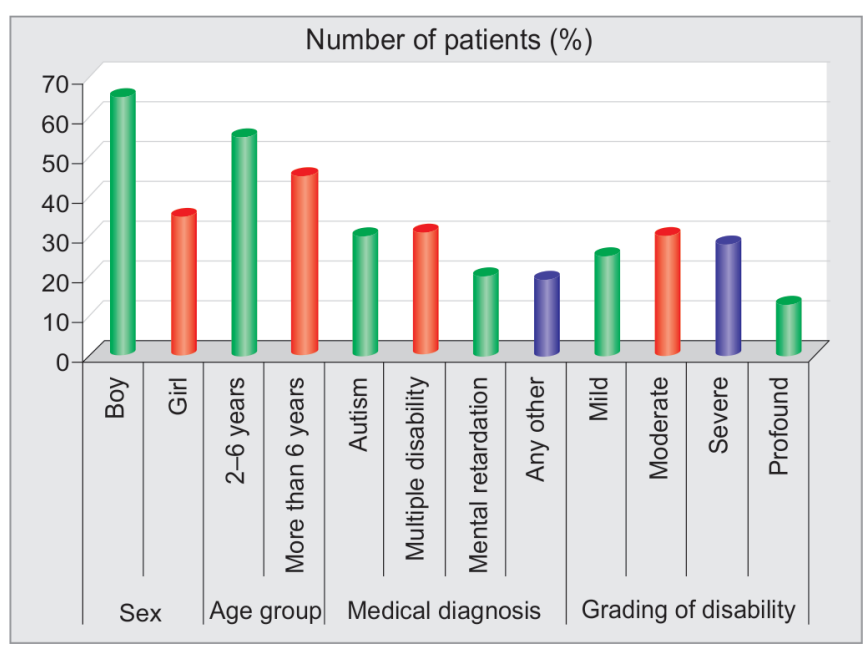

Graph 1: Demographic characteristics of the study population 
Table 1: Demographic distribution of DMF/DEF teeth indices

\begin{tabular}{llll}
\hline Demographic parameter & DEF/DMF teeth & $p$-value \\
\hline Sex & Boy & $11.48 \pm 4.74$ & $0.198 \mathrm{NS}$ \\
& Girl & $13.96 \pm 5.20$ & \\
Age group & 2-6 years & $14.01 \pm 4.41$ & $0.003 \mathrm{~S}$ \\
& More than 6 years & $10.96 \pm 5.22$ & \\
Medical & Autism & $12.52 \pm 3.90$ & $0.658 \mathrm{NS}$ \\
diagnosis & Multiple disability & $12.19 \pm 4.96$ & \\
& Mental retardation & $11.99 \pm 4.82$ & \\
& Any other & $13.59 \pm 4.90$ & \\
Grading of & Mild & $14.05 \pm 4.11$ & $0.512 \mathrm{NS}$ \\
disability & Moderate & $11.42 \pm 4.56$ & \\
& Severe & $12.30 \pm 5.02$ & \\
& Profound & $11.15 \pm 7.81$ & \\
\hline
\end{tabular}

NS: Nonsignificant; S: Significant

Table 3: Prevalence of malocclusion in subjects up to 12 years of age

\begin{tabular}{lllll}
\hline & $2-6$ & More than & No. of & p-value (Chi- \\
Parameter & years & 6 years & subjects & square test) \\
\hline Malocclusion & 16 & 59 & 75 & $0.004 \mathrm{~S}$ \\
No. of malocclusion & 28 & 7 & 35 & \\
\hline Total & 41 & 49 & 90 & \\
\hline
\end{tabular}

S: Significant

placement, as shown in Table 2. No significant difference was seen in patients undergoing pulpal therapy, composite restorations, and extractions. Table 3 shows the prevalence of malocclusion in subjects up to 12 years of age. A statistically significant result was observed in patients when divided based on their age ( $p$-value $<0.05)$.

\section{DISCUSSION}

Efficiency of dental treatments performed under general anesthesia is very high due to minimum cooperation required from the patient, and the fact that whole of the treatment can be done in a single sitting. Still, it is considered as last option because of certain health-dependent risk factors. Studies show that child's neurodevelopment is affected by general anesthesia administered in childhood. 6,7 The American Academy of Pediatric Dentistry has also standardized certain guidelines and indications for the use of general anesthesia in CSHCN. ${ }^{8}$ Hence, we assessed the oral health status of pediatric patients with
Table 2: Distribution of dental treatment and number of teeth treated by various treatment modalities divided based on the age group

\begin{tabular}{llll}
\hline $\begin{array}{l}\text { Dental treatment } \\
\text { modalities }\end{array}$ & 2-6 years & $\begin{array}{l}\text { More than } \\
6 \text { years }\end{array}$ & $p$-value \\
\hline $\begin{array}{l}\text { Pulpal therapy } \\
\text { Composite resin }\end{array}$ & $7.45 \pm 4.01$ & $3.48 \pm 3.53$ & $0.004 \mathrm{~S}$ \\
restoration & $7.01 \pm 3.96$ & $7.58 \pm 5.11$ & $0.845 \mathrm{NS}$ \\
$\begin{array}{l}\text { Complete crown } \\
\text { Extraction }\end{array}$ & $5.95 \pm 3.45$ & $2.45 \pm 3.08$ & $0.001 \mathrm{~S}$ \\
\hline Total & $1.48 \pm 2.50$ & $1.81 \pm 2.61$ & $0.425 \mathrm{NS}$ \\
\hline NS: Nonsignificant; S: Significant & &
\end{tabular}

special care needs undergoing dental treatment under general anesthesia.

In the present study, the mean DMF/DEF teeth index for children less than 6 years of age was 14.01 years, which is significantly higher as compared to the index in children with higher age group, as shown in Table 1. Similar results were reported by Chen et $\mathrm{al}_{1}{ }^{9}$ who observed a significant difference in the children of lower age group as compared to children of higher age. These results regarding the DMF/DEF teeth index might indicate that children receiving hospitalization are slightly more prone to poor oral health. While comparing the incidence of different treatment modalities in different age groups of CSHCN, we observed that statistically significant alterations were seen only in pulp therapy and complete crown procedures in different age groups as compared to all other dental treatment modalities (Table 2). Also, only crown procedures had significantly altered prevalence among children with autism, mental retardation, multiple disabilities, and other special health care needs as shown in Table 4. A significant alteration was seen in malocclusion incidence in different age groups (Table 3). Premature loss of teeth leading to the alteration in space in the dental arches was the most common cause of malocclusion observed in our study. Decaying teeth in CSHCN are often not treated on time, leading to complete destruction of teeth due to caries. Such teeth are treated by extracting them before their physiologic exfoliation time. This further leads to malocclusion. ${ }^{10-12}$

Savanheimo et $\mathrm{al}^{13}$ analyzed the various dental treatment modalities given under general anesthesia in the

Table 4: Distribution of dental treatment and number of teeth treated by various treatment modalities divided on the basis of type of disability

\begin{tabular}{llllll}
\hline Dental treatment modalities & Autism & Multiple disability & Mental retardation & Others & $p$-value \\
\hline Pulpal therapy & $6.59 \pm 3.84$ & $6.01 \pm 4.12$ & $5.45 \pm 4.19$ & $5.64 \pm 4.17$ & $0.845 \mathrm{NS}$ \\
Composite resin restoration & $5.42 \pm 3.81$ & $7.81 \pm 5.81$ & $7.52 \pm 4.31$ & $7.96 \pm 4.51$ & $0.351 \mathrm{NS}$ \\
Complete crown & $6.12 \pm 3.94$ & $4.16 \pm 3.51$ & $2.43 \pm 3.01$ & $3.82 \pm 3.26$ & $0.006 \mathrm{~S}$ \\
Extraction & $0.84 \pm 1.42$ & $1.10 \pm 1.92$ & $2.23 \pm 3.91$ & $2.08 \pm 2.70$ & $0.215 \mathrm{NS}$ \\
\hline Total & $14.76 \pm 3.51$ & $14.76 \pm 3.87$ & $13.75 \pm 4.97$ & $15.01 \pm 4.01$ & $0.628 \mathrm{NS}$ \\
\hline
\end{tabular}

NS: Nonsignificant; S: Significant 
Helsinki Public Dental Service. From the results, they concluded that the main reasons for the use of general anesthesia in the dental treatments were the extreme noncooperation, dental fear, and an excessive need for treatment. Personal and medical background and immigration status governed the factors, which should be taken into consideration while planning the dental treatments under general anesthesia. Savanheimo et $\mathrm{al}^{14}$ followed up the children receiving comprehensive dental care under general anesthesia. They prospectively analyzed the general healthy children treated with dental treatments under general anesthesia by Helsinki Public Dental Service in 2004 and concluded that in their study, generally, healthy children still require special attention due to their uncooperative nature due to dental fear. Dougherty ${ }^{15}$ reviewed the dental needs of special patients undergoing treatment under general anesthesia. He emphasized that general anesthesia can play a vital role in providing dental treatment for individuals who present with challenging behaviors. Literature still lacks sufficient significant research data regarding the same topic. Therefore, it totally relies on the dental practitioners and the pediatricians to have complete knowledge about the child's cognitive behavior and mental status. The decision of whether to take general anesthesia as a part of dental treatment for $\mathrm{CSHCN}$ is an art. Wang et $\mathrm{al}^{16}$ reviewed the importance in dental anesthesia in patients with special needs. Accordingly, they emphasized that general anesthesia is required in those pediatric patients who cannot bear the dental treatment. Reason for such rejection of dental treatment may be psychological, medical, or behavioral problems. Guidelines have been proposed by American Society of Anesthesiology (ASA) for office-based anesthesia for ambulatory surgeries. Therefore, the key for successful dental procedures is the harmony of relationship between dental surgeon and anesthesiologist.

\section{CONCLUSION}

From the above results, it can be concluded that in CSHCN dental problems and needs as well as caries indices are increased regardless of the type or extent of disability. A higher prevalence of malocclusion occurs in patients of comparatively older age and with fully developed dentition. Therefore, both the patient and their guardians should be motivated for pursuing regular dental check-up.

\section{REFERENCES}

1. American Academy of Pediatric Dentistry. Clinical guideline on management of persons with special health care needs. Pediatr Dent 2004;26:77-80.

2. Saghal J, Sood PB, Raju OS. A comparison of oral hygiene status and dental caries in children on long term liquid oral medications to those not administered with such medications. J Indian Soc Pedod Prev Dent 2002 Dec;20(4):144-151.

3. Leone CW, Oppenheim FG. Physical and chemical aspects of saliva as indicators of risk for dental caries in humans. J Dent Educ 2001 Oct;65(10):1054-1062.

4. Parry JA, Khan FA. Provision of dental care for medically compromised children in the UK by general dental practitioners. Int J Paediatr Dent 2000 Dec;10(4):322-327.

5. Lee PY, Chou MY, Chen YL, Chen LP, Wang CJ, Huang WH. Comprehensive dental treatment under general anesthesia in healthy and disabled children. Chang Gung Med J 2009 Nov-Dec;32(6):636-642.

6. Sun L. Early childhood general anaesthesia exposure and neurocognitive development. Br J Anaesth 2010 Dec;105 (Suppl 1): i61-i68.

7. Stratmann G. Review article: neurotoxicity of anesthetic drugs in the developing brain. Anesth Analg 2011 Nov;113(5): 1170-1179.

8. The American Academy of Pediatric Dentistry. Guideline on behaviour guidance for the pediatric dental patient; 2011. Available from: http://www.aapd.org.

9. Chen CY, Chen YW, Tsai TP, Shih WY. Oral health status of children with special health care needs receiving dental treatment under general anaesthesia at the dental clinic of Taipei Veterans General Hospital in Taiwan. J Chin Med Assoc 2014 Apr;77(4):198-202.

10. Lewis CW. Dental care and children with special health care needs: a population-based perspective. Acad Pediatr 2009 Nov-Dec;9(6):420-426.

11. Huang ST, Lin YS, Ho YT, Wang NT, Chung YM, Hsiao SY et al. Dental health status and treatment needs of elementary school children in Taiwan. J Dent Sci 2007;2(2):103-109.

12. Gussy MG, Waters EG, Walsh O, Kilpatrick NM. Early childhood caries: current evidence for aetiology and prevention. J Paediatr Child Health 2006 Jan-Feb;42(1-2):37-43.

13. Savanheimo N, Sundberg SA, Virtanen JI, Vehkalahti MM. Dental care and treatments provided under general anaesthesia in the Helsinki Public Dental Service. BMC Oral Health 2012 Oct 27;12:45.

14. Savanheimo N, Miira M Vehkalahti MM. Five-year follow-up of children receiving comprehensive dental care under general anaesthesia. BMC Oral Health 2014 Dec 15;14:154.

15. Dougherty N. The dental patient with special needs: a review of indications for treatment under general anaesthesia. Spec Care Dentist 2009 Jan-Feb;29(1):17-20.

16. Wang YC, Lin IH, Huang CH, Fan SZ. Dental anesthesia for patients with special needs. Acta Anaesthesiol Taiwan 2012 Sep;50(3):122-125. 